(Aus dem pharmak. Laboratorium der Universität St. Wladimir zu Kiew.

[Director: Prof. Dr. J. Laudenbach.])

\title{
Ueber ein Verfahren der manometrischen Registrirung der Zusammenziehungen des isolirten Säugethierherzens.
}

Von

\section{Dr. A. Siewert.}

(Mit 4 Textfiguren.)

Die Registrirung der Zusammenziehungen des isolirten Säugethierherzens folgt meistentheils durch Uebertragen derselben auf einen Schreibhebel (Engelmann), oder auf ein Luftkapselsystem (Langendorff) vermittelst eines mit einem Faden versehenen Häkchens. Die Autoren, sowie ihre Schüler u. A. (bei uns in Russland Kuljabko) erhielten dabei die besten Erfolge; es wäre dennoch wünschenswerth, diese Zusammenziehungen auch nach der manometrischen Methode registriren zu können, da man dabei nicht nur über den Rhythmus und die Energie der Zusammenziehungen, sondern auch über die geleistete Arbeit des Herzens, was bei Untersuchungen der Herzthätigkeit sehr wichtig ist, die besten Ergebnisse erzielt. Ausserdem hat die manometrische Methode der Registrirung noch den grossen Vorzug, dass man dabei nicht nöthig hat, sich des berussten Papiers zu bedienen, und dadurch die Möglichkeit erhält, die Registrirung des Herzens eine beliebig lange Zeit ununterbrochen zu verfolgen. Ich will hier noch auf den Umstand hinweisen, dass der intracardiale Druck, der ja ohne Zweifel im hohen Grade die Herzthätigkeit beeinflusst, bei den Verfahren von Engelmann und Langend orff vollständig ausfällt.

In Anbetracht dieser Vorzüge suchte ich auf Anregung des Herrn Prof. J. Laudenbach, die manometrische Methode zur Registrirung der Zusammenziehungen des isolirten Säugethierherzens in Anwendung zu bringen. 
Wie bereits bekannnt, kann die manometrische Methorle nur unter der Bedingung der beständigen Flüssigkeitsfüllung des Manometersystems ihre Anwendung finden. Diese Bedingung kann jedoch nicht erfüllt werden, da die Hoblräume des nach dem gebräuchlichen Verfahren von der Aorta aus gespeisten Herzens keine Flüssigkeit enthalten: der Zufluss derselben aus der Aorta in die linke Kammer wird durch die Aortenklappen verhindert, der Zufluss in die rechte aus derem Vorhofe, wohin die Flüssigkeit aus der Aorta durch die Coronargefässe gelangt, kann auch nicht zu Stande kommen in Folge des freien Abflusses der Flüssigkeit aus dem Vorhofe nach aussen durch die offenen hohlen Venen.

Diese Umstände können allerdings weder der Füllung des Manometersystems mit Flüssigkeit, noch letzterer Druckschwankungen Platz machen. Will man also die Manometermethode für die Registrirung des isolirten Herzens anwenden, so muss man entweder die gebräuchliche, von Langendorff angegebene Speisungsart ändern, indem man, statt von der Aorta, die Speisungsflüssigkeit von den Hohlräumen des Herzens zuleiten lässt, oder man sucht auf irgend eine andere Weise die Flüssigkeitsfüllung der Herzhohlräume zu ordnen.

Die Speisung des isolirten Herzens von dessen Hohlräumen aus kann nach dem von Pratt ${ }^{1}$, einem Schüler von Porter, beschriebenen Verfahren vorgenommen werden. Die Speisungsflüssigkeit wird dabei unter einem gewissen Drucke in den rechten Vorhof eingeleitet, von wo aus sie theils in die rechte Kammer und weiter durch die Art. pulmonalis nach aussen, theils aber in die Venulae Thebesii und von hier durch die Coronargefässe und Aorta ebenfalls nach aussen gelangt. Bei dieser Speisungsordnung des Herzens wird aber erstens die Herzwand auf rückläufigem Wege von den Venulae Thebesii und der V. coronaria aus ernährt, und zweitens ist die Durchflussgesehwindigkeit verhältnissmässig zu langsam, da hier der gebräuchliche Anfangsdruck der Durchströmungsflüssigkeit von 100-120 $\mathrm{mm} \mathrm{Hg}$ kaum zur Anwendung kommen kann. Dieser Umstände wegen verwarfen wir das Verfahren von Pratt und suchten nach einem anderen, der normalen Ernährungsweise des Herzens näher stehenden Verfahren.

Wir versuchten auf der Höhe der Thätigkeit des nach üblicher

1) Citirt nach O. Langendorff, Ergebnisse der Physiologie 1902. 
Weise von der Aorta aus gespeisten Herzens die Speisungslösung durch die Pulmonalvenen in den linken Vorhof zu leiten. Nach unserer Annahme sollte nun die Flüssigkeit in die linke Kammer und weiter in die Aorta gelangen, wo bei Regulirung des Abflusses in Folge Zusammenziehungen der Kammer ein gewisser Druck entstehen sollte, der die Durchspülung der Coronargefässe und folglich die Ernährung des Herzens bedingen könnte. Dieses Verfahren erfordert allerdings vom Herzen eine sehr energische, dem normal gespeisten, nicht isolirten Herzen gleiche Arbeit, was man a priori kaum erwarten konnte. Einige Versuche, die in dieser Hinsicht vorgenommen wurden, zeigten uns auf's Beste, dass die Energie des isolirten Herzens für dessen Selbsternährung nicht ausreiche: die kraftvollen Zusammenziehungen wurden beim Einleiten der Speisungsflüssigkeit in den linken Vorhof immer schwächer und verschwanden sogar; bei der Speisung von der Aorta aus traten sie wieder ein und nahmen an Kraft zu.

Auf diese Weise mussten wir den Versuch der Ernährung des Herzens von den Hohlräumen aus ganz aufgeben.

Aber auch bei der gebräuchlichen Ernährungsweise von der Aorta aus kann man die gewünschte Füllung der Hohlräume des Herzens und des Manometersystems leicht erzielen, indem man die Gefässe des rechten Vorhofes unterbindet.

Die Speisungslösung fliesst dabei aus der Aorta durch das Coronarsystem in den rechten Vorhof und in die rechte Kammer, von wo sie durch die Art. pulmonalis theils nach aussen, theils aber in das mit dieser Arterie verbundene Manometersystem gelangt. Die Zusammenziehungen des rechten Ventrikels erzeugen dabei im Manometersystem gewisse Druckschwankungen, die schon zur Registrirung der Herzthätigkeit angewandt werden können. Die rechte Kammer erscheint aber bei diesem Versuchsverfahren während der Diastole ungenügend gefüllt, da von den Coronarvenen aus verhältnissmässig wenig Flüssigkeit zufliesst. Man kann diesen Umstand nach zweierlei Verfahren vermeiden.

Nach dem ersten werden die Semilunarklappen der Art. pulmonalis ausgeschlossen durch Finbindung in die letztere einer Canüle, welche bis in den rechten Ventrikel gelangt. Die Flüssigkeit tritt dabei während der Systole aus dem Ventrikel in das Manometersystem, während der Diastole aber in Folge der Druckdifferenz, die jetzt im Manometersystem und im Ventrikel besteht, kehrt diese 
Flüssigkeit wieder in den Ventrikel zurück und bedingt damit die nöthige diastolische Füllung des Ventrikels.

Wir bevorzugten aber diesem Verfahren, bei dem ja der so zu sagen physiologische Strom der Flüssigkeit gestört wird, ein zweites, bei welchem man die nöthige Flüssigkeit, wie weiter beschrieben wird, direct in den rechten Vorhof durch die V. cava inferior aus einer speciellen Flasche hineinleiten lässt. Da nun auf diese Weise die beständige Füllung des Manometersystems mit Flüssigkeit und deren Druckschwankungen erzielt wurden, so konnte man schon die manometrische Registrirmethode in Anwendung bringen.

Allerdings können dabei nur die Zusammenziehungen der rechten Kammer registrirt werden. Dieser Umstand kann aber kaum den Werth des Verfahrens vermindern, da man ja meistentheils auch bei den Untersuchungen am nichtisolirten Herzen sich mit den Ergebnissen der Thätigkeit des einen, wenn auch des linken Ventrikels beschränkt. Die Methoden von Engelmann und Langendorff können in dieser Hinsicht kaum bevorzugt werden, da man dabei eigentlich nicht sagen kann, bei Thätigkeit welcher Kammer die erhaltenen Curven gewonnen sind. Die Registrirung der rechten Kammer hat andererseits am isolirten Herzen vor der linken den Vorzug, dass am isolirten Herzen die Energie der Thätigkeit der rechten Kammer oft viel stärker erscheint als die der linken; die rechte Kammer kann sich sogar stark und regelmässig zusammenziehen; während die linke sich kaum oder gar nicht bewegt, was bereits von $\mathrm{Gross}^{1}$ ), $\mathrm{Kuliabk0^{2 }}$ ) u. A. beobachtet wurde.

Zur Speisung des isolirten Säugethierherzens benutzen wir folgende, in der beigelegten schematischen Zeichnung dargestellte, Vorrichtungen, die im Allgemeinen nach der wohlbekannten, von Lang end orff angegebenen Methode construirt wurden.

Der Durchströmungsapparat besteht aus einem Druckgefäss und einem Flaschensystem, aus welchem die Speisungsflüssigkeit unter Druck von $100-120 \mathrm{~mm} \mathrm{Hg}$ durch die Ansehlusseanüle in die Aorta einfliesst und durch die Coronargefässe $u$. V. cava nach aussen gelangt. Der Druck in der Luftflasche wird beständig durch Zufluss von Wasser aus einem eirca 8-10 Liter fassenden mariotischen

1) Pflüger's Arch. Bd. 99 S. 264.

2) Ibid. Bd. 97 S. 539. 
Gefäss, welches sich auf einer dem nöthigen Drucke entsprechenden Höhe befindet, automatisch regulirt ${ }^{1}$ ).

Das Erwärmen der Speisungsflüssigkeit erfolgt bei unserer Versuchsanordnung zuerst in einem grossen mit Wasser gefüllten Gefäss, dessen Temperatur auf $38-40^{\circ} \mathrm{C}$. gehalten wird, und dann wieder in der Nähe des Herzens in einem Glasschlangenrohr, welches sich in einem besonderen Gefäss mit Wasser von $38-39^{\circ} \mathrm{C}$. befindet. Bei diesem Verfahren der Erwärmung wird erstens die Temperatur

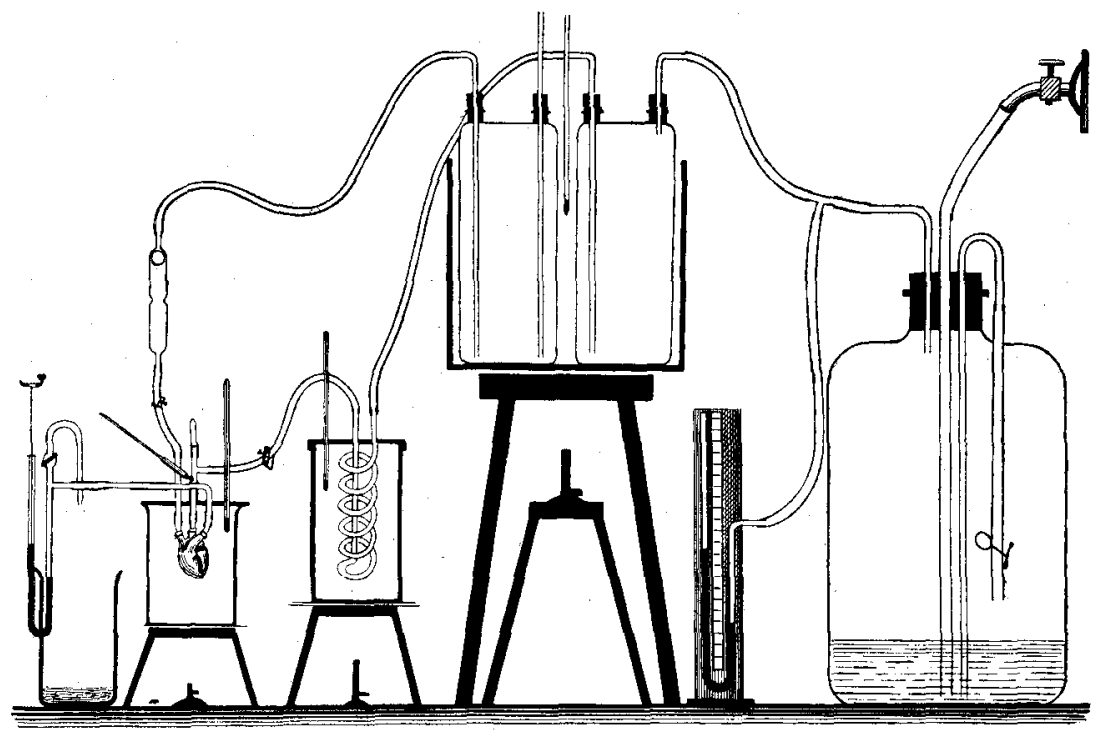

Fig. 1.

der in's Herz einfliessenden Flüssigkeit sehr wenig von der Durchflussgeschwindigkeit beeinflusst, was bei den Verfahren von Langendorff und besonders von Hering sehr leicht eintreten kann, und zweitens wird dabei das Herz der schädlichen Einwirkung der hohen Temperatur in Folge von Beschleunigung der Durchflussgeschwindigkeit vollständig entzogen, da die Temperatur der Wasserbäder höchstens auf $40^{\circ} \mathrm{C}$. gebracht wird. In dem grossen Wasserbade befindet sich ausser dem Flaschensystem mit Speisungslösung noch eine specielle mit Ringer's Salzlösung gefüllte mariotische Flasche, die mit dem Druckgefäss nicht in Verbindung steht. Das

1) Diese Druckregulirungsvorrichtung ist auf der Zeichnung nicht dargestellt. 
Wasserbad befindet sich in einer Höhe von $15-20 \mathrm{~cm}$ über der unteren Oeffnung der Anschlusscanüle. In Folge dessen fliesst die Salzlösung aus der Flasche unter einem beständigen Drucke von etwa $10-15 \mathrm{~mm} \mathrm{Hg}$ in den rechten Vorhof durch die untere Hohlvene, die mit dem Schlauch der Flasche verbunden wird. Um den Rückfluss der Salzlösung aus dem Vorhofe während der Systole zu verhindern, ist der Schlauch der Flasche mit einem nach Perles angefertigten Glasventil versehen. Dieser Schlauch ist noch mit einem Thermometer in der Nähe des Herzens versehen. (Auf der Zeichnung nicht dargestellt.)

Die Regulirung der zum Herzen zufliessenden Flüssigkeiten erfolgt mittelst $\mathrm{H}$ of fmann's Schraubklemmen. -

Das Herz befindet sich während des Versuches in einer Wärmekammer, die aus einem Zinkblechdeckel und einem grossen Reagensglase besteht. Der Zinkdeckel ist an der Anschlusscanüle, die durch das Centrum des Deckels führt, befestigt. Das Reagensglas wird, nachdem das Herz in Verbindung mit dem Apparate gebracht ist, untergestellt und durch eine kleine Gasflamme erwärmt. Ausser der Oeffnung für die Anschlusscanüle befinden sich im Deckel noch Oeffnungen für ein Thermometer und für die beiden Schläuche des Manometers und der speciellen Flasche.

Die Registrirung der Herzschläge erfolgt mittelst eines Manometers von Boehm. Durch die mit einem Hahn versehene Oeffnung am oberen Ende des absteigenden Rohres des Manometers fliesst bei unserer Versuchsanordnung die Flüssigkeit aus dem Herzen nach aussen. Als Speisungsfiüssigkeit diente uns meistentheils die von Locke angegebene Salzlösung. Die Speisungsflüssigkeit und das Druckgefäss werden mit Sauerstoff aus dem Bunzen'schen Gasometer nach Möglichkeit gesättigt.

Der Versuch wird auf folgende Weise ausgeführt. Nach Freilegung des Herzens des verbluteten Thieres werden die obere Hohlvene und die Gefässe des Aortenbogens unterbunden. Die Aorta und V. cava inferior werden mit Glascanülen versehen. Die Lungen werden nach Unterbindung der Wurzeln abgeschnitten. Das Herz wird ausgeschnitten und nach Befreiung von Blutgerinnseln mit dem Apparate in Verbindung gebracht. Man lässt Locke'sche Salzlösung durchfliessen, wobei das Herz bald zu schlagen anfängt. Währenddem wird die Art. pulmonalis freigeiegt, mit einer Canüle versehen und mit dem Manometerschlauch verbunden. 


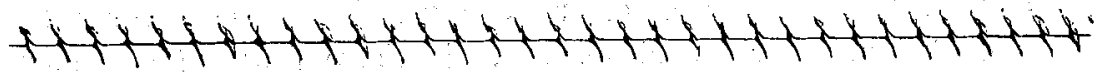
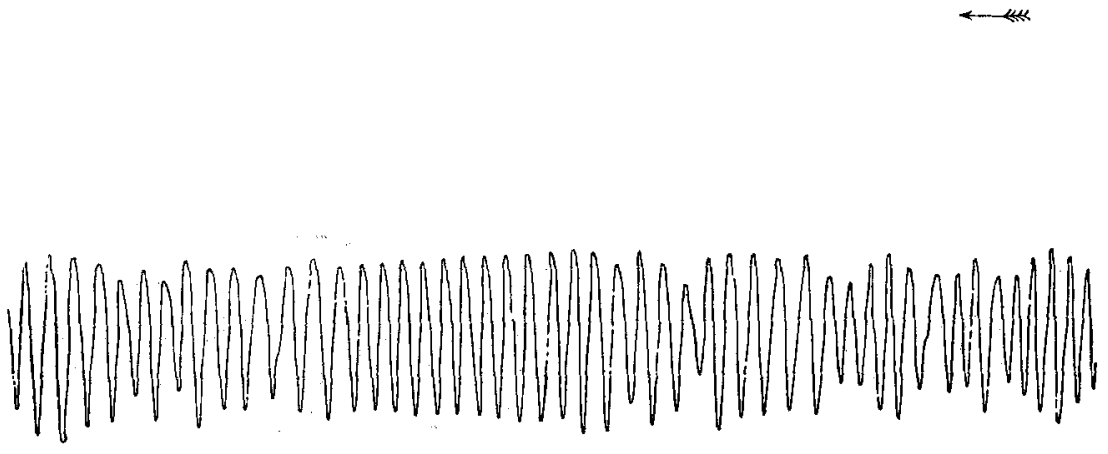

Fig. 2. Katzenherz. Bei niedrigem Drucke in der Art. pulmonalis. Locke's Salzlösung. (Von rechts nach links zu lesen.)
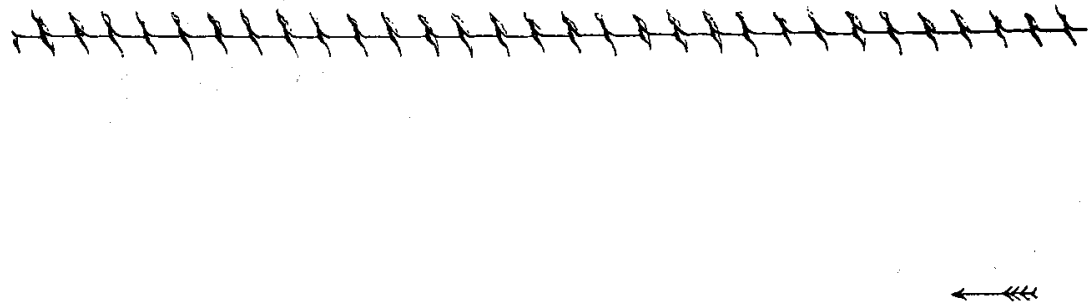

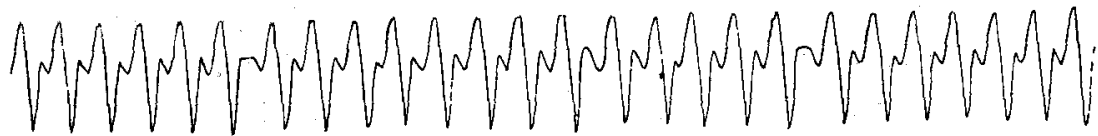

Fig. 3. Dasselbe Herz. Bei höherem Drucke in der Art. pulmonalis. L o cke's Salzlösung. 
Ueber ein Verfahren der manometrischen Registrirung etc.
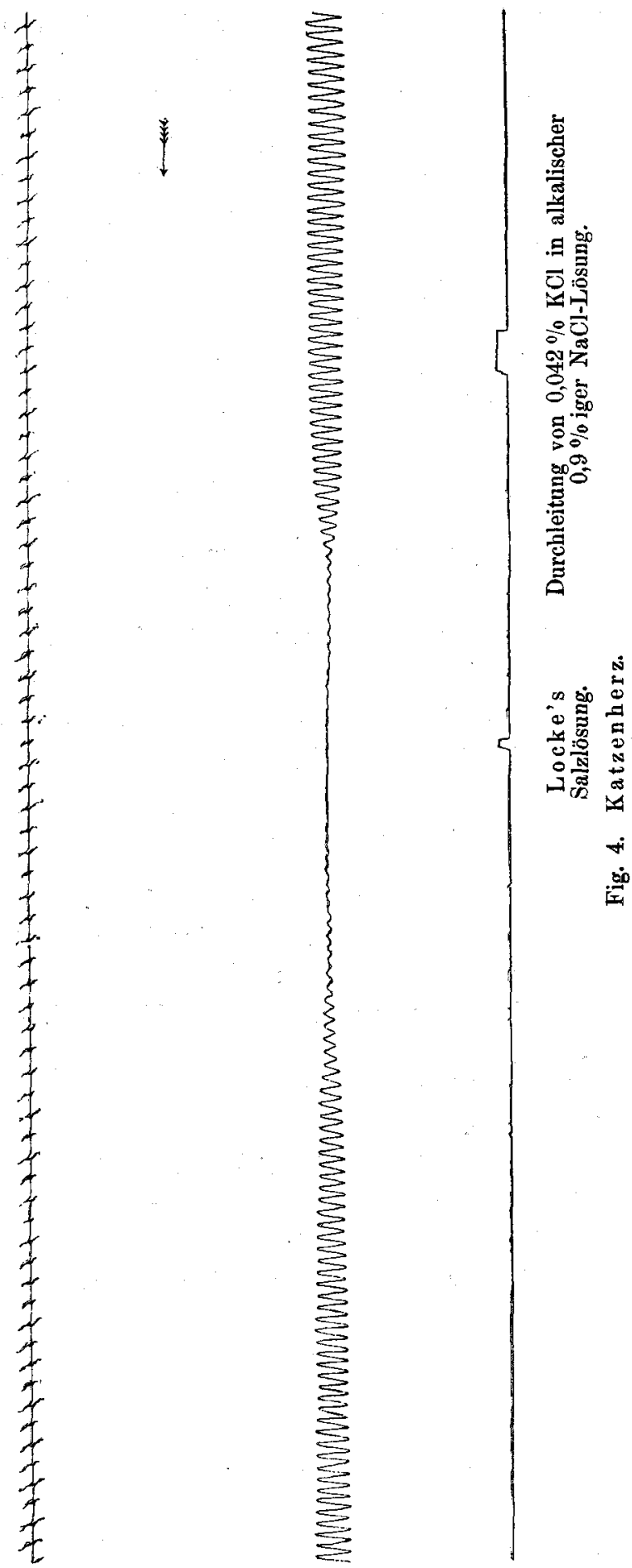
Die Flüssigkeit fliesst dabei aus der Aorta durch die Kranzgefässe in den'rechten Vorhof, von wo sie durch die untere Hohlvene nach aussen gelangt. - Nach Eintreffen der regelmässigen Zusammenziehungen wird der Manometerhahn geöffnet und die untere Hohlvene mit dem Schlauche der speciellen Flasche verbunden, wonach das Herz in die Wärmekammer eingeschlossen wird. -

Jetzt fliesst die Flüssigkeit aus dem rechten Vorhof in die Kanmer und gelangt durch die Art. pulmonalis in das Manometersystem und durch dessen Oeffnung nach aussen. -

Regulirt man nun den Zufluss aus der speciellen Flasche und den Abfluss aus der Manometeröffnung, so erhält man in Folge der Zusammenziehungen der rechten Kammer regelmässige Schwankungen des Quecksilbers im Manometer, die diese Zusammenziehungen recht gut registriren.

Als graphische Beispiele führe ich hier einige Curven bei, die an Katzenherzen gewonnen worden sind. -

Diese Methode der Registrirung bietet keinerlei Schwierigkeit; dieselbe erlaubt uns nicht nur die Contractionen des normal gespeisten, sondern auch des unter verschiedenen Einflüssen stehenden Herzens lange Zeit ununterbrochen zu registriren. -

\section{Nachtrag bei der Correctur.}

Bei weiteren Versuchen hat es sich erwiesen, dass das Herz sich stets vollkommener contrahirte, wenn wir folgende Veränderungen in der Versuchsanordnung anstellten:

1. Den Druck in der speciellen Flasche bis auf $1,5-2 \mathrm{~mm} \mathrm{Hg}$ verminderten.

2. Das Glasventil von Perles durch ein Bunsen'sches Gummiventil ersetzten.

Ausserdem fanden wir die Function der Semilunarklappen der Art. pulm. sehr oft ungenügend und ersetzten dieselben durch ein zartes Bunsen'sches Gummiventil am Manometerschlauche; die Canüle wird dabei direct in den Ventrikel eingeführt. 